\title{
Robotics in Autism Intervention
}

\author{
https://doi.org/10.3991/ijes.v7i4.11448 \\ Polyxeni Ntaountaki, Georgia Lorentzou, Andriana Lykothanasi, \\ Panagiota Anagnostopoulou, Vasiliki Alexandropoulou, \\ Athanasios S. Drigas $(\bowtie)$ \\ N.C.S.R. 'Demokritos', Institute of Informatics and Telecommunications, \\ Telecoms Lab, Net Media Lab, Athens, Greece \\ drait.demokritos.gr
}

\begin{abstract}
Autism Spectrum Disorders is a group of lifelong disabilities that affect people's communication and underlying social messages. The state of the art is an indication of how technology, and in particular robotics, can offer promising tools to enhance research and treatment in ASD. This review represents an attempt to investigate how robot-assistive therapy strategies help children with autism increase social interaction and to imitate, recognize and express feelings. One of the major questions of this paper was if the robots appear to be effective means in assistive therapies. The research team of this paper concluded that robots are discovered to be a predictable and secure environment for ASD children and to be quite efficient in the intervention process. In addition to the above-mentioned, this project also broaches ethical issues that should be taken into consideration by the researchers and therapists during humanrobot interplay.
\end{abstract}

Keywords-Robots, autism, intervention

\section{Introduction}

Autism is a state involving a large variety of disorders with a weakening of social relations, communication and imagination, as well as seriousness and the nature of the symptoms varies from one person to another. Autism is a pervasive developmental disorder since it affects the person during all the periods of his development. The characterization "pervasive" indicates that the disorder globally affects the development of the person and the term "disorder" expresses the sense of deviation from normal. Autism has no cure but with early intervention, much can be done to improve the quality of life of those who have been affected. Several therapeutic approaches are in such a state during the treatment years. However, due to the nature of the disorder and its large variety of symptoms, there cannot be a single approach established as the best treatment model, because it can work well with a child but may not work at all with another $[1,2]$.

The use of robots in autism intervention has been widely used in the last years. Over the last decade, robots are used as intervention tools for individuals with Autism 
Spectrum Disorder (ASD). There are many robots with different appearances and features which imitate the movements, the body language and the facial expressions of humans. Robots have been used as assisting technology in many areas of autism research. In particular, they have been used to help children engage in social processes, to teach complex social behaviors, such as personal space recognition, adaptability, control communication and recognition of emotion. Generally, robots offer incredible capabilities to monitor and influence the behavior of autistic children as well as to help the therapist. However, it is worth mentioning that their cost is very high and its efficient use requires being monitored by a technician $[3,4]$.

In this paper, we present researches on robots concerning the therapy of children with autism. First, we present robots that enhance the social skills of children with autism as social interaction is a predominant feature in autism. Then we refer to robots related to the empowerment of emotion of children with autism through imitation and other activities. Finally, we discuss the ethical issue of using therapeutic robots as an intervention process in autism. The main purpose of this paper is to investigate how robots positively or negatively influence the intervention of autism in the area of social and emotional interaction.

\section{Social Skills Improvement}

According to Sartorato F., Przybylowski L. \& Sarko D. K., [4] during the last decade, robots have been used as intervention tools for individuals with Autism Spectrum Disorder (ASD). There are many robots with different appearances and features which imitate the movements, the body language and the facial expressions of humans. The AuRoRa project presented the robot "ROBOTA" which helped children with ASD develop their social skills and their joint attention and imitate the movements of the robot. Moreover, another humanoid robot whose name is KASPAR helped children with ASD learn how to collaborate with adults, by enhancing their social behavior, too. The first humanoid robot, KASPAR, was developed and prototyped in 2005 by M. Blow as a Human Robot Interaction (HRI). Since then, the robotic platform has continuous development due to the needs of the users and the technological advancements [5]. Further information about KASPAR is reported at the end of this section.

Additionally, robots with a cartoonish appearance, whose name, are Tito and Keepon, respectively were attractive to children with ASD and helped them imitate facial expressions and increase their joint attention. Also, robots that looked like animals were used as intervention tools for children with ASD and helped them develop new social skills and imitate positive behaviors. Examples of this kind of robots are PABI which can imitate human emotions and Probo which can tell social stories to children with ASD and teach the appropriate behavior according to social conditions. Research has proved that robot-assisted therapy helped children with ASD generalize skills taught during the intervention in real interaction with adults and children. Moreover, this kind of therapy increased the levels of interaction and children with ASD developed their social skills even more [4]. 
Before almost ten years, Stanton et al. indicated the intimacy between children and robots. Initially, they investigated the interactions between four children with autism and a humanoid robot named Robota. Two of the four children showed an increase in all sessions in the overall level of interaction with the robot. Then they examined 11 children with autism using an autonomous dog-like robot (AIBO) compared to Kasha, a mechanical dog, and they concluded that the children in the AIBO room spent more time interacting with AIBO than with KASHA. In addition, they used more words per minute with AIBO. They dealt more often with authentic interaction with the experimenter when they had AIBO. In the interaction with AIBO, social interaction was increased, a behavior associated with children of a typical development. From the indicative results it was found that robots can help children with autism in social interaction [6].

Six years later, another pilot research by Barakova et al. referred to LEGO therapy based on human-robot interaction with children within the spectrum of autism. The humanoid robot replaces one of the children in the game scenario that follows the design of LEGO therapy. By using long training scenarios, children keep their interest during training. A pilot and final experiment was designed, executed and analyzed where a robot intervened in the LEGO treatment between fairs of children mediated by a robot during the course, with the aim of completing an overall LEGO construction. Barakova et al. came up with two findings: a) play-based robot scenario in which play continues during sessions opened up possibilities for long-range interventions using robots and led to a semantic increase in social initiations during the intervention in natural settings; b) including dyadic interactions between a robot and a child within triadic games with robots has positive effects on the child's engagement and on creating learning moments that conform to the chosen therapeutic approach [7].

In the same year, Salter et al. introduced the Queball robot, which has a simple global morphology combined with a collection of autonomous behaviors. The platform is simple, secure, and provides a wide range of kinetic, virtual, audio and tactile interactive features aimed at enhancing the child's learning and playing abilities. It also provides connectivity to other devices or to a computer via WiFi. The results showed that this device can be easily used in schools, without any special technical support. The interaction between the child with autism and the robot was characterized as calm and interesting, because the child seemed relaxed and entertained. Finally, it was observed that children with autism developed the ability to play with other people without facing any conflicts [8].

Furthermore, Tennyson et al. described the fourth generation of robot agents using public platforms, the Lego NXT, as Socially Assistive Robotics (SAR). The above platform is combined with pedagogical guidelines and social scenarios in order to support an alternative educational approach to learning social behavior. Robot social assistants (SARs) allow children to interact with a three-dimensional object through touch, verbal communication, physical play and enables them to learn through imitation and interaction, thus encouraging autonomous social behavior. The results showed that SARs have helped to reduce the stereotypic behavior of the individuals compared to the results of human interaction [9]. 
A study was performed by Srinivasan et al. in which children with ASD were divided into 3 groups in a random manner to accept the appropriate intervention. The rhythm group took part in activities with movement and music, the robot group in activities whose purpose was to learn how to imitate robot games and the comparison group focused on typical activities on a tablet screen. The results of this study showed that the robot group reached higher scores when they expressed themselves. The participants of the robot group didn't spend enough time communicating with the robot, but they developed their social verbalization levels and their language skills during the sessions. Nevertheless, the children with ASD of the robot group couldn't generalize learned skills in a real social interaction with a human. Due to the restricted motor movement, the limited and difficult wordiness and the partial autonomy of the robots, there were many disadvantages in robot-assisted long-term interventions for children with ASD [10].

Another research that supports the use of robots in autism intervention is the one by Esteban et al. where Robot-Assisted Therapy (RAT) is presented. The survey was divided into two phases. In the first phase, a robot called Wizard of Oz (WoZ) was used, while in the second phase he RET robots were used under a semiautomatic system. When the two phases were completed, they were compared with Standard Human Treatment (SHT). The results showed that some of the participants had a better performance while interacting with robots and it was concluded that RAT had effectually been used to refine social skills in children with ASD [11].

According to Desideri et al. in a study with a sample of three preschool children diagnosed with ASD, the NAO robot was used as an intervention method. The intervention consisted of two parts (control and robot intervention) in which children participated in activities using objects and toys of their everyday life and there were different targets such as imitation, communication or language comprehension, as well as positive feedback. In both parts, activities had the same structure, but the only difference was that the children interacted with the educator in the first session and with the robot in the second. The results showed that the robot played an important role in the intervention and helped children maintain their attention and achieve their goals in the activities. The most important result was that the children participated more actively in the interaction with the robot than with the educator [12]. In addition, Hina et al. focused on the interaction of autistic individuals with NAO which differs from the rest in its methodology, because it uses a multimodal fusion of information and has the ability to interpret a complex command and divide it into elementary work on basics for interaction. As a result, the NAO robot makes the social interaction for ASD children easier [13].

Furthermore, Taheria, Meghdaria, Alemia and Pouretemad developed an innovative robotic music therapy intervention, originally designed to teach the basic principles of how to play the drums and the xylophone to children with autism, using the NAO robot as an assistant and then help them develop their social and cognitive skills as well as their kinetic imagination. The aim of the study was to evaluate the results of the interventions of the three groups associated with the program. At first, the robot or parent or therapist would playing music through real drums or xylophone and the child would have to do the same. The NAO robot was programmed to play the musi- 
cal instruments with the baguettes placed in its hands. The results of the survey proved that the social and cognitive skills of the participants in the study were positively influenced. However, in general, the effects of music therapy on participants' behavior appeared to be different for children who belonged to different degrees of the spectrum [14].

In a recent study Scassellati et al. introduced an intervention in which 12 children diagnosed with ASD took part and attended special education lessons. The participants interacted both with a social autonomous robot and their caregiver for half an hour on a daily basis for a month. At the beginning of each session, the robot told a story of children's everyday life and then the children played games guided by the robot. The purpose of the intervention was to adopt the participants' different social skills and generalize them while interacting with adults. The results of this study proved that the robot helped children develop their social and communicative skills. Moreover, the participants paid more attention when there was a robot than when they played a game themselves. The most important result was that the children were able to adapt these social skills in real interactions with adults [15].

Melo et al. presented the INSIDE system, a network-based robot system designed to allow the use of mobile robots as active players in the treatment of children with autitic disorders. The system consisted of an autonomous mobile robot, ASTRO, which was able to engage in social interaction during a health care session as the child moved around the room while completing various activities. The robot played a key role in the healing process, as it was the role of the robot to invite the child to perform the various activities, explain the activities to the child and provide encouragement. This work pioneered the use of an autonomous robot of driving and multitasking skills, acting as a social factor and engaging in rich social interaction with fully autonomous children. The INSIDE system stands out from other systems, because it allows for a complex, semi-structured interaction in ASD therapy, while fully autonomous robots are used [16].

Moreover, an important way for robots to intervene in autism is their contribution to the process of finding a job. Kumazaki et al. developed a work environment simulation tutorial, using an Android Robot and looked at changes in self-confidence in learning non-verbal communication skills and in reducing stress. These changes were measured through personal reports of participants about their confidence level and the levels of cortisol in their saliva. The results of the above measurements showed that the question about whether or not they wanted to re-interview the participants through the robot was answered with a "yes". Also, in the robot team, they all completed the test without any signs of discomfort or technological worries. Still, the results showed that in the non-verbal communication category there was a significant improvement for the robot team [17].

Finally, a long term study about KASPAR showed that it can be used to foster and support collaborative play among children with autism. Six children with autism took part in controlled play sessions both with and without the robot, using a designed collaborative game that was based on imitation. The study demonstrated how the different pairs of children with autism improved social behaviors in playing with each other after they had played in pairs with the robot KASPAR [18]. In addition, a recent 
study by Wood et al. proved that KASPAR can help children with autism develop their visual perspective skills (the ability to see the world from another person's perspective [19].

Nevertheless, Alhaddad et al. offered another aspect to social robots' interaction and examined the harm caused by the interaction between social robots and children with autism, especially during their provocative behaviors, that is, when they throw objects, kick, or beat themselves up. These behaviors can harm both the children and those around them. So, they quantified the magnitude of this damage, based on severity indicators for one of the provocative behaviors, namely the throwing of objects. The results showed that total injury levels based on selected severity markers are relatively low compared to the respective limits [20].

\section{Improvement of Emotional Abilities}

In addition to the social empowerment of robots in autism therapy, robots have also helped improve the emotional abilities of autistic children who, as it is known, are facing difficulties. According to Adams and Robinson, children with ASD have a tendency towards feeling comfortable with non-humanoid robots. Nevertheless, a robot with real human features helps children with ASD develop facial recognition skills. The researchers refer to the FACE project (Facial Automation for Conveying Emotions), which uses a realistic android head and its main purpose is to enhance the social and emotional skills of children. The android head is able to illustrate six basic emotions and can be controlled by the therapist and the child. During the intervention, the behavior of the participants, their interaction with the android head and the imitation of the facial expressions were observed. The results showed that there can be an increase in the recognition of realistic emotions [21]. Additionally, Pop et al. used the social robot Probo to investigate if it can help children with autism identify situationbased emotions. The results of their work showed that the performance of the participants improved. In particular, children's performance improved with moderate to large effect sizes in identifying both sadness and happiness. So, this study proved that robot-assisted therapy can improve not only the social but also the emotional ability of children with autism [22].

Moreover, Leo et al. study was to become the first attempt to use machine learning strategies during ASD child-robot interactions in terms of mimicking facial expressions, making it possible to objectively assess children's behavior and then introduce a measure of treatment effectiveness. In particular, the program focused on basic emotional recognition skills. In addition to the above, the applied innovations can also contributed to this work by introducing a Facial Expression Recognition (FER) mechanism that can automatically detect and monitor the face of the child and then recognize emotions based on machine learning. Two different experimental sessions took place: the first examined the FER with datasets capable of presenting the proposed action and assigning the existing precision of recognition strategies. The second was ASD children and it was a preliminary research into who can use the introduction of 
the FER engine in the therapeutic protocol that was effectively used to monitor children's behavior [23].

Salvador, Silver, and Mahoor conducted a study using the humanoid Zeno Robot. The aim of the study was to compare the emotion recognition of children with and without ASD. Firstly, the robot communicated with the children and then Zeno presented to the children six basic emotions (happiness, surprise, sadness, anger, disgust and fear) through a game. The participants made a guess for each emotion and the robot evaluated the answer if it was true or false. At the end of the game, the children could interact with the robot. The results showed that there was no difference between the two groups in recognizing emotions. Children with autism found it difficult to distinguish Fear or Disgust, but both groups made better scores when the emotions were presented through gestures [24].

The following year, in Boccanfuso et al. study, an emotion-simulating robot was used in order for child-robot interactions and affective responses to this robot to be analyzed. In this way, not only were differences between the responses of typically developing children and children with autism examined, but also play and affective response and its connection to the severity of autism calibrated using Autism Diagnostic Observation Schedule (ADOS) was tested [25].

The research by Costa et al. aims to improve the emotional capacity of children with autism through education, using the QT robot with a screen on its face where emotions are displayed visually with the help of animated characters. Emotions are expressed in a simple way and there are different levels of difficulty. During the process, emotional and social situations are presented, using paradigms in order to be comprehended by the children [26].

A study conducted by So et al. had as a target to teach Chinese children with ASD the recognition and the production of pantomime gestures that could help them express their feelings using the NAO robot. The intervention contained two phases: to recognize gestures and to produce them. The children participating in the study were separated into two groups: the control group and the intervention group. The results of this study proved that the children were taught to recognize and produce gestures while interacting with the NAO robot. On the other hand, the participants weren't able to correctly produce gestures when they communicated with a human and they couldn't generalize the meaning of gestures in real human interaction [27].

Chevalier et al. designed a personalized robot environment for social learning for people with autism. They assessed the possible relationship between recognition expressions of the body/face and emotions as well as proprietary and visual concepts of integrating an atom. Firstly, they described the design of EMBODIEMO a database platform containing video body/face feelings and emotions. Then, they examined the relationship between recognition of emotions and visual profiles of people with typical development (TD) and people with ASD. As expected, results showed that people who were focused more on visual indications had the best recognition scores. However, they found that TD individuals focused on eloquence had better recognition results. In particular, participants with ASD got lower emotion recognition scores. According to the results, the combination of visual and perceptive signals in autism in- 
tervention is very important, because they can affect children with ASD's ability in recognizing emotions [28].

Javed et al. presented a new robot-based intervention framework, designed to target the aesthetic difficulties of cure and emotional therapy in children with autism. Three types of systems are used to provide expandable robotic interactions with socioemotional expressions based on gestures and characters. The framework of intervention includes an emotional interaction game in mobile computing environments, an interactive robotic encounter with social scenarios and gesture recognition games that measures emotional processing and verbal skills at a socio-emotional level. They created two groups. The findings showed that children with ASD participated less in the emotion control game and had less focus on appearance but had more average cases of self-diagnosed interactions and imitation robot behaviors. While their overall performance in the gesture recognition game was lower than the control group, it should be noted that the best individual performance came from an ASD child [29].

Another recent research by Marino et al. studied a semi-autonomous robot. In this study, 14 children participated randomly divided into two groups (control group and experimental group). A semi-autonomous robot was used that had the potential to offer support, efforts to communicate and express feelings and information about the participants' behavior through a camera. The findings showed that both groups (control and experimental) before the intervention had lower performance than expected given their age. It is important to note that the performance of both teams improved after the intervention, but the children interacting with the robot showed a greater difference in understanding the emotions. In addition, there was an improvement in understanding the views, feelings, and thoughts of others, where autistic people proved to be deficient [30].

\section{$4 \quad$ Ethical Issues}

There are many ethical issues that should be taken into consideration when implementing humanoid robots in autism-related robot-assisted therapy (RAT) such as the level of emotional attachment, the use of robots as human substitutes in therapy and the need for briefing the target group on the standard ethical guidelines and protocols. More specifically, autistic children can believe that the robot is an autonomous, independent being that is able to interact and attach in an intelligent way. An approach, where the child clearly perceives the robot as a friendly educational toy instead of a substitute for a human friend, can be the solution to the problem. As far as the use of the robot as a human is concerned, results showed that the majority of the participants agree to include robots in the therapy because it is ethically acceptable and only 26 per cent believe that robots can replace humans in the therapy. Lastly, the participants should give their consent before joining the experiment and be briefed on the ethical protocol and guidelines [31].

Moreover, Mark Coeckelbergh et al. have conducted a survey of questions about what people think of using robots in ASD therapy. The results are the most important for some of the basic ethical and therapeutic issues identified when it comes to robots. 
The analysis is based on the answers of two questions, "Is it ethically accepted for social robots to used in treatment for children with autism?" and "Is it ethically accepted for social robots to be used in health care?". The vast majority of respondents agreed in using robots in the health care system, including robot-assisted treatment for ASD children. However, according to researchers' discussion of ethical issues, there has been considerable number of participants (44\%) opposed the idea of robots replacing the therapists. On the contrary, many respondents preferred that interaction to be supervised by the therapist and for the robot to operating teleoperated way rather than in a fully automated way [32].

The Haring et al. article also mentions examples of robotic negative effects on the ethical aspect. Submissions are intended to be out forward and can take a wide variety of views (including analytical, empirical, technical, or planning-oriented perspectives) but are expected to be well established in humanoid robot intervention (HRI) and technology ethics literature. Such examples are inverted robots (e.g., malicious robots, robots that provide information to inadvertent sources), misleading robot presentation (e.g. people's trend to provide sensitive information to robots, a trend of people to undertake automation work), dark applications (e.g. psychological manipulation of people by robots, responses to the application of robot rules), unintended consequences of social robots (e.g. a disadvantage of explanatory robots, unintentional robot operations as a threat, socially interactive consequences of a robot, impact of robots on interpersonal relationships), solutions (e.g., enforcement of good practices, giving to the man tools to defeat the dark side, public education strategies for informing users about real concerns when it comes to social robots) [33].

Concerning the ethical design of the robotic systems and application with robots, there are also legal initiatives, such as the ISO 13482:2014 Personal Care Robots, the BS 8611:2016 Guide and IEEE Ethically Aligned Design 2017 from the IEEE Global Initiative and Standard Association, which refer to the positive and negative effects of robot technology. Moreover, there are international policies on drones and driverless cars and specific laws, which have to do some disorders such as autism. Nevertheless, these initiatives give unclear instructions to the people who work with robots [34].

According to Villaronga \& Albo-Canals, a social robot is both a technological product and a social being. This combination makes it difficult to understand what legal requirement must be followed when the researchers design a social robot intervention. The only ethical issue about the design of robots is ISO13482:2014, which specify robots' characteristics such as the robot shape, the robot movements, energy supply and storage and autonomous decisions of the robot. Moreover, social robots interact with special needs children and develop a relationship with them. In the EU the General Data Protection Regulation is legislated in May 2018 and the personal information of the users in therapies with robots must be protected. Hence, the design of social robots should follow and obey the rules, so as not to put at risk the therapy of people with special needs, such as children with ASD [34]. 


\section{Conclusion}

Considering all the above, it is clear that robot-based therapies can help with the treatment of children with autism. All the robots which have been presented in this paper have been designed to take up a lot of roles through engaging activities in order to educate children with autism in the social and emotional field. Imitation, joint attention, emotion and facial recognition, triadic interaction and tactile social behaviour were examined during the above mentioned studies. In conclusion, the social skills and emotional improvement children with ASD demonstrate while interacting with robots, allow us to accept robots' important role in autism therapy. However, there are some ethical issues that should be taken into consideration, such as the emotional attachment between children and robots and the percentage of uses in the whole treatment process. Overall, we strongly believe that humans should not be replaced with robots in autism therapy, despite their beneficial role robotics technology should be rather a supportive tool operated by humans.

\section{References}

[1] M.E Block, V.E Block, and P. Halliday, "What is autism?" Teaching Elementary Physical Education, vol. 17, no. 6, pp 7-11, November 2006.

[2] A. Gena, Autism and pervasive developmental disorders. Athens: Idiotiki. 2002.

[3] M. Sharmin, M.M. Hossain, A. Saha, M. Das, M. Maxwell and S. Ahmed, "From Research to Practice: Informing the Design of Autism Support Smart Technology," In Proc. of the 2018 CHI Conference on Human Factors in Computing Systems, no. 102, 2018, pp. 1-16. https://doi.org/10.1145/3173574.3173676

[4] F. Sartorato, L. Przybylowski and D.K. Sarko, "Improving therapeutic outcomes in autism spectrum disorders: Enhancing social communication and sensory processing through the use of interactive robots," Journal of Psychiatric Research, vol .90, pp. 111,July,2017.Available:https://www.sciencedirect.com/science/article/abs/pii/S00223 95616306562. [Accessed: 25 May, 2019]. https://doi.org/10.1016/j.jpsychires.2017. 02.004

[5] L.J. Wood, A. Zaraki, M.L. Walters, O. Novanda, B. Robins and K. Dautenhahn, "The iterative development of the humanoid robot kaspar: An assistive robot for children with autism," In Proc. of the International Conference on Social Robotics, 2017, pp. 53-63. https://doi.org/10.1007/978-3-319-70022-9 6

[6] C.M. Stanton, P.H. Kahn, R.L. Severson, J.H. Ruckert and B.T. Gill, "Robotic animals might aid in the social development of children with autism," In Proc. of 3rd ACM/IEEE International Conference on Human-Robot Interaction (HRI), 2008, pp. 271-278. https://doi.org/10.1145/1349822.1349858

[7] E.I. Barakova, P. Bajracharya, M. Willemsen, T. Lourens and B. Huskens, "Longterm LEGO therapy with humanoid robot for children with ASD," Expert Systems, vol. 32, no. 6, pp. 698-709, November, 2014. Available:https://onlinelibrary.wiley. com/doi/full/10.1111/exsy.12098. [Accessed: 18 June, 2019]. https://doi.org/10. $\underline{1111 / \text { exsy. } 12098}$

[8] T. Salter, N. Davey and F. Michaud, "Designing and developing Queball, a robotic device for autism therapy," In Proc. of the 23rd IEEE International Symposium on 
Robot and Human Interactive Communication, 2014, pp. 574-579. https:// doi.org/10.1109/roman.2014.6926314

[9] M.F. Tennyson, D.A. Kuester, J. Casteel and C. Nikolopoulos, "Accessible Robots for improving social skills of individuals with autism," Journal of Artificial Intelligence and Soft Computing Research, vol. 6, no. 4, pp. 267-277, August, 2016. Available: https://www.degruyter.com/downloadpdf/j/jaiscr.2016.6.issue-4/jaiscr-20160020/jaiscr-2016-0020.pdf [Accessed: 24 May, 2019]. https://doi.org/10.1515/jaiscr2016-0020

[10] S.M. Srinivasan, I.M. Eigsti, T. Gifford and A.N. Bhat, "The effects of embodied rhythm and robotic interventions on the spontaneous and responsive verbal communication skills of children with Autism Spectrum Disorder (ASD): A further outcome of a pilot randomized controlled trial," Research in Autism Spectrum Disorders, vol. 27, pp. 73-87, July, 2016. Available: https://www.sciencedirect.com/science/article/ pii/S1750946716300435. [Accessed: 22 May, 2019]. https://doi.org/10.1016/j.rasd. $\underline{2016.04 .001}$

[11] P.G. Esteban, P. Baxter, T. Belpaeme, E. Billing, H. Cai, H.L. Cao, M. Coeckelbergh, C. Costescu, D. David, A.D. Beir, Y. Fang, Z. Ju, J. Kennedy, H. Liu, A. Mazel, A. Pandey, K. Richardson, E. Senft, S. Thill, G.V. de Perre, B. Vanderborght, D. Vernon, H. Yu and T. Ziemke, "How to build a supervised autonomous system for Robot-Enhanced therapy for children with autism spectrum disorder," Paladyn, Journal of Behavioral Robotics, vol. 8, no. 1, pp. 18-38, May, 2017. Available: https:// www.degruyter.com/view/j/pjbr.2017.8.issue-1/pjbr-2017-0002/pjbr-2017-0002.xml [Accessed: 15 May,2019]. https://doi.org/10.1515/pjbr-2017-0002

[12] L. Desideri, M. Negrini, M.C. Cutrone, A. Rouame, M. Malavasi, E.J. Hoogerwerf, P. Bonifacci and R. Di Sarro, "Exploring the Use of a Humanoid Robot to Engage Children with Autism Spectrum Disorder (ASD)," In Proc. of Association for the Advancements of Assistive Technology (AAATE) Conference, 2017, pp. 501-509. https://doi.org/10.31234/osf.io/3fvr6

[13] M.D. Hina, A.R. Cherif and G. Ivanova, "Robotic Interaction for Assistance to Autistic Children," In Proc. of the 19th International Conference on Computer Systems and Technologies, 2018, pp. 178-184. https://doi.org/10.1145/3274005.3274026

[14] A. Taheria, A. Meghdaria, M. Alemia and H.R. Pouretemad, "Teaching music to children with autism: A social robotics challenge," Scientia Iranica, vol.26, no.1, pp. 40-58, February, 2019. Available: https://www.researchgate.net/publication/3313 03709 Teaching music to children with autism A social robotics challenge. [Accessed: 18 May, 2019]. https://doi.org/10.24200/sci.2017.4608

[15] B. Scasselati, L. Boccanfuso, C. M. Huang, M. Mademtzi, M. Qin, N. Salomons, N. Ventola and F. Shic, "Improving social skills in children with ASD using a long-term, in-home social robot," Science Robotics, vol. 3, no. 21, pp. 1-9, August, 2018. Available: https://robotics.sciencemag.org/content/3/21/eaat7544.short. [Accessed: 2 June,2019]. https://doi.org/10.1126/scirobotics.aat7544

[16] F.S. Melo, A. Sardinha, D. Belo, M. Couto, M. Faria, A. Farias, H. Gambôa, C. Jesus, M. Kinarullathil, P. Lima, L. Luz, A. Mateus, I. Melo, P. Moreno, D. Osório, A. Paiva, J. Pimentel, J. Rodrigues, R. Ventura, "Project INSIDE: towards autonomous semi-unstructured human-robot social interaction in autism therapy," Artificial Intelligence in Medicine, vol. 96, pp. 198-206, May, 2019. Available: https://www. sciencedirect.com/science/article/pii/S0933365717305997\#!. [Accessed: 25 June,20 19]. https://doi.org/10.1016/j.artmed.2018.12.003 
[17] H. Kumazaki, T. Muramatsu, Y. Yoshikawa, B.A. Corbett, Y. Matsumoto, H. Higashida, T. Yuhi1, H. Ishiguro, M. Mimura and M. Kikuchi, "Job interview training targeting nonverbal communication using an android robot for individuals with autism spectrum disorder," SAGE Journals, vol.23, no. 6, pp. 1586-1595, August, 2019. Available:https://journals.sagepub.com/doi/full/10.1177/1362361319827134.

[Accessed: 20 May, 2019]. https://doi.org/10.1177/1362361319827134

[18] J. Wainer, B. Robins, F. Amirabdollahian and K. Dautenhahn, "Using the humanoid robot KASPAR to autonomously play triadic games and facilitate collaborative play among children with autism," IEEE Transactions on Autonomous Mental Development, vol. 6, no. 3, pp. 183-199, April, 2014. Available: https://ieexplore. iee.org/abstract/document/6784461. [Accessed: 8 June,2019]. https://doi.org/10. $1109 /$ tamd.2014.2303116

[19] L.J. Wood, B. Robins, G. Lakatos, D.S. Syrdal, A. Zaraki and K. Dautenhahn, "Developing a protocol and experimental setup for using a humanoid robot to assist children with autism to develop visual perspective taking skills," Paladyn, Journal of Behavioral Robotics, vol. 10, no. 1, pp 167-179, March, 2019. Available: https://www.degruyter.com/view/j/pjbr.2019.10.issue-1/pjbr-2019-0013/pjbr-20190013.xml. [Accessed: 16 June,2019]. https://doi.org/10.1515/pjbr-2019-0013

[20] A.Y. Alhaddad, J.J. Cabibihan and A. Bonarini, "Head Impact Severity Measures for Small Social Robots Thrown During Meltdown in Autism," International Journal of Social Robotics. vol.11, no. 2, pp. 255-270, April, 2019. Available: https://link.spri nger.com/article/10.1007/s12369-018-0494-3. [Accessed: 15 May,2019]. https://doi. org/10.1007/s12369-018-0494-3

[21] A. Adams and P. Robinson, "An Android Head for Social-Emotional Intervention for Children with Autism Spectrum Conditions," In: D’Mello S. et al. (eds) Affective Computing and Intelligent Interaction. ACII 2011. Lecture Notes in Computer science, vol. 6975, pp. 183-190, 2011. Available: https://link.springer.com/chapter/ 10.1007/978-3-642-24571-8 19 [Accessed: 2 June,2019]. https://doi.org/10.1007/ 978-3-642-24571-8_19

[22] C.A. Pop, R. Simut, S. Pintea, J. Saldien, A.S. Rusu, D. David, J. Vanderfaeillie, D. Lefeber and B. Vanderborght, "Can the social robot Probo help children with autism to identify situation-based emotions? A series of single case experiments," International Journal of Humanoid Robotics, vol. 10, no. 3, pp. 1350025/2- 1350025/24, August,2013.Available: https://www.researchgate.net/publication/263879288 Can the social robot Probo help children with autism to identify situation based emotions A series of single case experiments. [Accessed: 15 June,2019]. https://doi.org/10.1142/s0219843613500254

[23] M. Leo, M.D. Coco, P. Carcagni, C. Distante, M. Bernava, G. Pioggia, G. Palestra, "Automatic Emotion Recognition in Robot-Children Interaction for ASD Treatment," In Proc. of the International Conference on Computer Vision Workshop (ICCVW), 2015, pp. 145-153. https://doi.org/10.1109/iccvw.2015.76

[24] M.J. Salvador, S. Silver, M.H. Mahoor, "An emotion recognition comparative study of autistic and typically-developing children using the zeno robot," In Proc. of the 2015 IEEE International Conference on Robotics and Automation (ICRA), 2015, pp. 6128-6133. https://doi.org/10.1109/icra.2015.7140059

[25] L. Boccanfuso, E. Barney, C. Foster, Y.A. Ahn, K. Chawarska, B. Scassellati and F. Shic, "Emotional robot to examine different play patterns and affective responses of children with and without ASD," In Proc. of the 11th ACM/IEEE international Con- 
ference on human- robot interaction (HRI), 2016, pp. 19-26. https://doi.org/10. $1109 /$ hri.2016.7451729

[26] A.P. Costa, G. Steffgen, F.R. Lera and A. Nazarikhorram, "Socially assistive robots for teaching emotional abilities to children with autism spectrum disorder," In Proc. of the $3^{\text {rd }}$ Workshop on Child-Robot Interaction at Human Robot Interaction (HRI), 2017.

[27] W. C. So, M.K.Y. Wong, C.K.Y. Lam, W.Y. Lam, A.T.F. Chui, T.L. Lee, H.M. Ng, C.H. Chan and D.C.W. Fok, 'Using a social robot to teach gestural recognition and production in children with autism spectrum disorders," Disability and Rehabilitation: Assistive Technology, vol. 13, no. 6, pp. 527-539, July, 2017. Available: https://www.tandfonline.com/doi/abs/10.1080/17483107.2017.1344886. [Accessed: 4 June,2019]. https://doi.org/10.1080/17483107.2017.1344886

[28] P. Chevalier, J.C. Martin, B. Isableu, C. Bazile and A. Tapus, "Impact of sensory preferences of individuals with autism on the recognition of emotions expressed by two robots, an avatar, and a human," Autonomous Robots, vol. 41, no.3, pp. 613-635, March, 2017. Available: https://link.springer.com/article/10.1007/s10514-016-9575z. [Accessed: 17 June, 2019]. https://doi.org/10.1007/s10514-016-9575-z

[29] H. Javed, M. Jeon, A. Howard and C.H. Park, "Robot-Assisted Socio-Emotional Intervention Framework for Children with Autism Spectrum Disorder," In Proc. of the $2018 \mathrm{ACM} / \mathrm{IEEE}$ International Conference on Human-Robot Interaction-HRI, 2018, pp. 131-132. https://doi.org/10.1145/3173386.3177082

[30] F. Marino, P. Chilà, S.T. Sfrazzetto, C. Carrozza, I. Crimi, C. Failla, M. Busà, G. Bernava, G. Tartarisco, D. Vagni, L. Ruta and G. Pioggia, "Outcomes of a RobotAssisted Social-Emotional Understanding Intervention for Young Children with Autism Spectrum Disorders," Journal of Autism and Developmental Disorders, pp. 1-15, March, 2019. Available: https://link.springer.com/article/10.1007/s10803-019-03953x. [Accessed: 18 May, 2019]. https://doi.org/10.1007/s10803-019-03953-x

[31] A. Tanevska, N. Ackovska and V. Kirandzisk, "Robot-assisted therapy: considering the social and ethical aspects when working with autistic children," In Proc. of the 9th International Workshop on Human-Friendly Robotics (HFR 2016), 2016, pp. 57-60.

[32] M. Coeckelbergh, C. Pop, R. Simut, A. Peca, S. Pintea, D. David and B. Vanderborght, "A Survey of Expectations About the Role of Robots in Robot-Assisted Therapy for Children with ASD: Ethical Acceptability, Trust, Sociability, Appearance, and Attachment," Science and Engineering Ethics, vol. 22, no.1, pp. 47-65, April, 2015. Available: https://link.springer.com/article/10.1007/s11948-015-9649-x. [Accessed: 22 June,2019]. https://doi.org/10.1007/s11948-015-9649-x

[33] K.S. Haring, M. Novitzky, P. Robinette, E. de Visser, A. Wagner and T. Williams, "The Dark Side of Human-Robot Interaction: Ethical Considerations and Community Guidelines for the Field of HRI," In Proc. of the 14th ACM/IEEE International Conference on Human-Robot Interaction (HRI), 2019, pp. 689-690. https://doi.org/10. 1109/hri.2019.8673184

[34] E.F. Villaronga and J. Albo-Canals, "I'll take care of you," said the robot. Reflecting up on the legal and ethical aspects of the use and development of social robots for therapy," Paladyn.Journal of behavioral robotics, vol.10, no. 1, pp. 77-93, January, 2019. Avaiilable:https://www.degruyter.com/view/j/pjbr.2019.10.issue-1/pjbr-20190006/pjbr-2019-0006.xml. [Accessed: 22 May,2019]. https://doi.org/10.1515/pjbr$\underline{2019-0006}$ 


\section{$7 \quad$ Authors}

Polyxeni Ntaountaki is with N.C.S.R. 'Demokritos', IIT Net Media Lab \& Mind

Brain R\&D, and a post graduate student in Special Educational Needs and ICTs at the Democritus University of Thrace (xeniantaountaki45@gmail.com).

Georgia Lorentzou is with N.C.S.R. 'Demokritos', IIT Net Media Lab \& Mind

Brain R\&D, and a post graduate student in Special Educational Needs and ICTs at the Democritus University of Thrace (georgialor@gmail.com).

Andriana Lykothanasi is with N.C.S.R. 'Demokritos', IIT Net Media Lab \& Mind Brain R\&D, and a post graduate student in Special Educational Needs and ICTs at the Democritus University of Thrace (lykothanasi.andriana@gmail.com).

Panagiota Anagnostopoulou is with N.C.S.R. 'Demokritos', IIT Net Media Lab \& Mind Brain R\&D, and a post graduate student in Special Educational Needs and ICTs at the Democritus University of Thrace (italgiota@gmail.com).

Vasiliki Alexandropoulou is with N.C.S.R. 'Demokritos'IIT Net Media Lab \& Mind Brain R\&D, and a post graduate student in Special Educational Needs and ICTs at the Democritus University of Thrace (vas.alex.1994@gmail.com).

Athanasios Drigas is Research Director at N.C.S.R. 'Demokritos', Institute of Informatics \& Telecoms Net Media Lab, Mind Brain R\&D, Agia Paraskevi, Athens, Greece (dr@iit.demokritos.gr).

Article submitted 2019-08-05. Resubmitted 2019-09-21. Final acceptance 2019-09-21. Final version published as submitted by the authors. 